\title{
HIV cure strategies: a complex approach for a complicated viral reservoir?
}

\author{
Matthew D Marsden ${ }^{1}$ \& Jerome A Zack*,1,2 \\ ${ }^{1}$ Department of Medicine, Division of Hematology \& Oncology, The David Geffen School of Medicine at the University of \\ California, Los Angeles, CA 90095, USA \\ ${ }^{2}$ Department of Microbiology, Immunology, \& Molecular Genetics, University of California, Los Angeles, CA 90095, USA \\ *Author for correspondence: Tel.: +1 310825 0876; Fax: +1 310267 1875; jzack@ucla.edu
}

\begin{abstract}
"One approach toward reservoir depletion is therefore to essentially speed up this process of viral production to force expression of the entire latent viral load"
\end{abstract}

First draft submitted: 28 November 2018; Accepted for publication: 29 November 2018; Published online: 13 December 2018

Keywords: AIDS • block and lock • cure • HIV • kick and kill

Human immunodeficiency virus (HIV) has exacted a terrible toll on individuals and populations around the world. By 2017 this virus had already resulted in the death of $\sim 35$ million people and a further estimated 37 million people were living with HIV at that time. Of these individuals, $\sim 60 \%$ were accessing antiretroviral therapy (ART), lifesaving drugs that inhibit virus replication and prevent progression to AIDS [1]. However, HIV is a retrovirus that infects long lived $\mathrm{CD}^{+} \mathrm{T}$ cells and permanently integrates into their chromosomal DNA. Integrated HIV also sometimes pauses its replication cycle to remain in a nonexpressing latent state [2]. These latently infected cells can persist for life, are not directly affected by antiretroviral drugs, and are not visible to the immune system [3]. Therefore, ART alone does not cure the infection but is instead administered on a daily basis to continuously prevent latent HIV from replicating again and causing disease. Thus, the 'latent reservoir' of HIV is a critical barrier to HIV cure.

\section{Transplantation approaches to create an HIV-resistant immune system}

A bone marrow transplant to repopulate the hematopoietic system with HIV-resistant cells is one strategy for HIV cure that appears to have been successful in the single case of the 'Berlin Patient' [4]. A substantial scientific effort is currently directed toward repeating this exciting success and improving on the broad strategy that led to it. Yet this type of transplantation procedure (which requires aggressive chemotherapy to clear space for the transplanted cells) is associated with significant health risks for the patient and is both expensive and dependent upon sophisticated medical infrastructure, making it currently unfeasible for most HIV-infected individuals across the world. Therefore, several additional and conceptually distinct efforts to eliminate latent HIV are nevertheless being pursued.

\section{'Kick \& kill' to flush out latent HIV}

HIV latency is problematic because infectious virus can be slowly released from the latent reservoir for the lifespan of the host. One approach toward reservoir depletion is therefore to essentially speed up this process of viral production to force expression of the entire latent viral load, allowing the host cells to be killed by viral cytopathic effects or the antiviral immune response, in a comparatively short space of time. This has variously been called an 'activation elimination' or 'kick and kill' approach [2]. Methods to achieve this have primarily focused on the identification or development of small molecule latency reversing agents (LRAs), which can induce expression of latent HIV. This has yielded some positive results using in vitro [5-7] and animal models for HIV latency [8], where quite robust HIV expression can be induced from latently infected cells in some cases. Early human studies [9] have also shown that certain classes of LRA can be safely administered [5] but this work is at a relatively early proof of concept phase and substantial effects on the size of the latent HIV reservoir in these human trials has not yet been achieved. 
Modifications of this kick and kill concept include augmenting the anti-HIV immune response around the time of viral reactivation, for example through passive transfusion of anti-HIV natural killer cells or cytotoxic-T lymphocytes, or vaccination with HIV antigens to boost the host's own immune response. Given the successful use of chimeric antigen receptor expressing cytotoxic-T lymphocytes in treatment of cancer [10], a similar strategy is also being explored to treat HIV, whereby genetic modification of immune cells is performed to arm them more effectively to kill HIV-expressing cells that have been stimulated from latency to produce HIV proteins [5]. The use of HIV-specific broadly neutralizing antibodies or immunotoxins may serve a similar purpose of enhancing the killing of latently infected cells, particularly if the level of HIV expression induced by an LRA is not sufficient to induce cell death via viral cytopathic effects. A complementary approach might include also 'priming' cells during latency reversal to make them more susceptible to apoptosis induced by HIV expression or immune effector mechanisms [11].

One potential limitation of kick and kill approaches is that they will have to induce expression of all the replication-competent HIV in the latent reservoir that might eventually lead to viral rebound. This may prove challenging because not all cells containing apparently intact HIV proviral genomes are induced to produce infectious virus when stimulated [12]. In some cases, the number of cells harboring apparently intact HIV genomes is 60 -fold higher than the number that produce infectious virus following a single-round of ex vivo stimulation. It might therefore be predicted that a single round of in vivo stimulation would also not be able to induce expression of all latent virus. This may perhaps be circumvented by repeated rounds of stimulation, with the same or different LRAs, that sequentially induce expression and depletion of infected cells in a stepwise fashion until the reservoir is eliminated. Alternatively, a kick and kill approach might deplete the fraction of the HIV reservoir that is relatively easy to activate but may not be able to induce expression of proviruses that are in a more deeply latent state at the time of LRA administration. Factors that might affect HIV expression from latency are complex and may include the specific integration site of the latent provirus, epigenetic constraints governing transcription such as histone acetylation or methylation patterns, or other physiologic factors in the host cell including HIV transcription factor availability [13], which together could contribute to a nonuniform or synchronous response to an LRA stimulus. Consequently, additional strategies toward HIV reservoir depletion are actively being pursued that are not reliant on inducing HIV expression.

\section{Genome editing to disrupt latent HIV proviruses}

Directly editing or excising integrated HIV proviruses would circumvent the problems associated with inducing HIV expression in all latently infected cells and would allow the latent HIV reservoir to be eliminated in situ, without the need for augmented immune responses. Therefore, efforts to edit HIV proviruses through evolved recombinases, zinc finger nucleases, CRISPR/Cas9 approaches and other methods are all being pursued [14-16]. Challenges with this type of approach largely center on issues of delivering the required proteins or nucleic acids to all HIV reservoir cells in the body. Strategies that require expression of proteins in the target latently infected cells face additional problems because resting $\mathrm{CD} 4^{+} \mathrm{T}$ cells are refractory to lentiviral vector transduction and also, have notoriously low metabolic activity and thus do not efficiently express exogenous proteins. In addition, if some latent HIV proviruses are present in inaccessible chromatin structures then the editing enzymes may not successfully access and inactivate all latent proviruses.

\section{'Block and lock' to lock-in latent HIV genomes \& prevent expression}

A more recent approach toward managing the latent reservoir is sometimes called 'block and lock' and is almost diametrically opposed to the 'kick and kill' paradigm $[17,18]$. Block and lock approaches seek to trap the latent proviruses into a permanently nonexpressing state. This may be performed with drugs that need to be taken on a continuous basis, which would be conceptually similar to current ART but may afford additional benefits by preventing release of virus from the latent reservoir that can contribute to the elevated immune activation observed in many individuals on long-term ART. Alternatively, if expression of the latent virus can be permanently silenced without the need for further drug treatment then a functional cure might be produced in this way. A substantial portion $(\sim 5-8 \%)$ of the human genome is already made up of sequences that are derived from retroviruses that can either no longer express or cannot make infectious virions [19,20]. A successful 'block and lock' approach may therefore turn HIV DNA into another harmless endogenous retroviral passenger in the human genome that is incapable of expressing proteins or spreading to new individuals. Block and lock approaches toward HIV cure are still at early developmental stages and are associated with some of their own challenges. For example, unlike the 
kick and kill or direct proviral disruption/editing approaches, with a block and lock strategy the individual will still harbor intact HIV genomes that encode replication-competent virus, which could in principal re-emerge at any point. However, this situation may not be so different from other HIV cure approaches. Even if the goal is to achieve a sterilizing cure and eliminate all replication-competent HIV from the body, ongoing monitoring will be needed to ensure that HIV hiding in a rogue cell somewhere in the individual does not emerge and rekindle infection many years after therapy is stopped.

\section{A complex cure for a complex reservoir?}

The above approaches are not mutually exclusive. For example, one could envisage a situation where potent ART suppresses ongoing virus replication and reduces overall HIV burden in the body. A series of 'kick and kill' doses could then eliminate latently infected cells that harbor easily inducible virus, and subsequent administration of 'block and lock' agents may lock the remaining proviruses into a permanently nonexpressing state. The risk of rebound may be further reduced by infusion of HIV-specific chimeric antigen receptor T cells, broadly neutralizing anti-HIV antibodies, or other methods to augment anti-HIV immunity, that can rapidly clear any rare infected cells that start producing virus. This type of combination approach may actually be more likely than a single-agent cure, since the complex and nuanced nature of the HIV latent reservoir may require an equally multifactorial and layered solution.

\section{Disclosure}

JA Zack is on the scientific advisory board for Bryologyx, and a co-founder of Protect Therapeutics.

\section{Financial \& competing interests disclosure}

The authors laboratories are funded by grant numbers Al131294 and Al124743 to JA Zack, Al124763 to MD Marsden and AI28697 (the UCLA Center for AIDS Research) from the National Institutes of Health. The authors have no other relevant affiliations or financial involvement with any organization or entity with a financial interest in or financial conflict with the subject matter or materials discussed in the manuscript apart from those disclosed.

No writing assistance was utilized in the production of this manuscript.

\section{Open access}

This work is licensed under the Attribution-NonCommercial-NoDerivatives 4.0 Unported License. To view a copy of this license, visit http://creativecommons.org/licenses/by-nc-nd/4.0/

\section{References}

1. Unaids. UNAIDS Global HIV \& AIDS statistics — 2018 fact sheet (2018). www.unaids.org/en/resources/fact-sheet

2. Marsden MD, Zack JA. HIV/AIDS eradication. Bioorg. Med. Chem. Lett. 23(14), 4003-4010 (2013).

3. Finzi D, Hermankova M, Pierson T et al. Identification of a reservoir for HIV-1 in patients on highly active antiretroviral therapy. Science 278(5341), 1295-1300 (1997).

4. Hutter G, Nowak D, Mossner M et al. Long-term control of HIV by CCR5 Delta32/Delta32 stem-cell transplantation. N. Engl. J. Med. 360(7), 692-698 (2009).

5. Marsden MD, Zack JA. Experimental approaches for eliminating latent HIV. For. Immunopathol. Dis. Therap. 6(1-2), 91-99 (2015).

6. Marsden MD, Wu X, Navab SM et al. Characterization of designed, synthetically accessible bryostatin analog HIV latency reversing agents. Virology 520, 83-93 (2018).

7. Pache L, Dutra MS, Spivak AM et al. BIRC2/cIAP1 Is a negative regulator of HIV-1 transcription and can be targeted by smac mimetics to promote reversal of viral latency. Cell Host Microbe 18(3), 345-353 (2015).

8. Marsden MD, Loy BA, Wu X et al. In vivo activation of latent HIV with a synthetic bryostatin analog effects both latent cell 'kick' and 'kill' in strategy for virus eradication. PLoS pathog. 13(9), e1006575 (2017).

9. Archin NM, Liberty AL, Kashuba AD et al. Administration of vorinostat disrupts HIV-1 latency in patients on antiretroviral therapy. Nature 487(7408), 482-485 (2012).

10. Maldini CR, Ellis GI, Riley JL. CAR T cells for infection, autoimmunity and allotransplantation. Nat. Rev. Immunol. 18(10), 605-616 (2018).

11. Cummins NW, Sainski AM, Dai H et al. Prime, shock, and kill: priming CD4 T cells from HIV patients with a BCL-2 antagonist before HIV reactivation reduces HIV reservoir size. J. Virol. 90(8), 4032-4048 (2016). 
12. Ho YC, Shan L, Hosmane NN et al. Replication-competent noninduced proviruses in the latent reservoir increase barrier to HIV-1 cure. Cell 155(3), 540-551 (2013).

13. Van Lint C, Bouchat S, Marcello A. HIV-1 transcription and latency: an update. Retrovirology 10, 67 (2013).

14. Zhu W, Lei R, Le Duff Y et al. The CRISPR/Cas9 system inactivates latent HIV-1 proviral DNA. Retrovirology 12, 22 (2015).

15. Qu X, Wang P, Ding D et al. Zinc-finger-nucleases mediate specific and efficient excision of HIV-1 proviral DNA from infected and latently infected human T cells. Nucleic Acids Res. 41(16), 7771-7782 (2013).

16. Karpinski J, Hauber I, Chemnitz J et al. Directed evolution of a recombinase that excises the provirus of most HIV-1 primary isolates with high specificity. Nature Biotechnol. 34(4), 401-409 (2016).

17. Mousseau G, Kessing CF, Fromentin R, Trautmann L, Chomont N, Valente ST. The tat inhibitor didehydro-cortistatin A prevents HIV-1 reactivation from latency. MBio 6(4), e00465 (2015).

18. Ahlenstiel C, Mendez C, Lim ST et al. Novel RNA duplex locks HIV-1 in a latent state via chromatin-mediated transcriptional silencing. Mol. Ther. Nucleic Acids 4(10), e261 (2015).

19. Marchi E, Kanapin A, Magiorkinis G, Belshaw R. Unfixed endogenous retroviral insertions in the human population. J. Virol. 88(17), 9529-9537 (2014)

20. Griffiths DJ. Endogenous retroviruses in the human genome sequence. Genome Biol. 2(6), REVIEWS1017 (2001). 